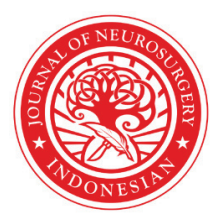

\title{
Epidermoid cyst of the cerebellopontine angle
} accompanied by trigeminal neuralgia: A rare case report

\author{
Agus Budi Setiawan' ${ }^{1}$ Lathif Suryandana ${ }^{2 *}$
}

\section{ABSTRACT}

Background: Trigeminal Neuralgia is a common hemifacial, paroxysmal shock-like pain caused by any disturbance at the fifth cranial nerve. The diagnosis is clinically plausible due to its specific symptoms. Almost an accepted scenario occurs from vascular compression on the root entry zone of the trigeminal nerve. As a clinical sign of intracranial tumors from epidermoid cyst or cholesteatoma, trigeminal neuralgia is a rare condition. A lesion, accompanied by an epidermoid cyst, constitute less than $1 \%$ of all intracranial space-occupying lesion.

Case Presentation: A 47-year-old female was referred to our center due to an increasing radiated pain level throughout the right face. The pain was characterized as a sudden, sharpshooting pain that lasted only for a few seconds. The episodes of pain occurred very often and triggered by simple activities, such as teeth brushing. Her symptoms had been initially controlled by carbamazepine but worsened, despite the fact that a higher dosage of carbamazepine and amitriptyline had been prescribed. Physical examination revealed a normal motoric function of the trigeminal nerve, whereas a brain MRI showed a lesion at the right cerebellopontine angle. This lesion was abutting and compressing the fifth cranial nerve root. The patient was treated micro-surgically and the lesion was excised by retrosigmoid approach. The histological report indicated epidermoid cyst or cholesteatoma. During the follow-up period, the patient recovered without any post-operative neurological deficits and, most notably, no further hemifacial pain reports.

Conclusion: Epidermoid tumors rarely cause trigeminal neuralgia but had been understood to cause trigeminal nerve neuropathy by either compressing the nerve directly or eliciting an inflammatory reaction. Our study found that the trigeminal nerve's decompression surgery by the total excision of the compressing tumor leads to a satisfactory result.
${ }^{1}$ Neurosurgeon, Head of Neurosurgical Department, Margono Soekarjo Hospital, Central Java, Indonesia ${ }^{2}$ Medical Doctor, Intern of Neurosurgical Department, Margono Soekarjo Hospital, Central Java, Indonesia

*Corresponding to: Lathif Suryandana; Medical Doctor, Intern of Neurosurgical Department, Margono Soekarjo Hospital, Central Java, Indonesia; lathif.suryandana@gmail.com

Received: 2020-08-21

Accepted: 2020-10-30 Published: 2020-11-23

Keywords: Trigeminal Neuralgia, Cerebellopontine Angle, Epidermoid Cyst, Pain

Cite This Article: Setiawan, A.B., Suryandana, L. 2020. Epidermoid cyst of the cerebellopontine angle accompanied by trigeminal neuralgia: A rare case report. Indonesian Journal of Neurosurgery 3(3): 84-87. D0I: 10.15562/ijn.v3i3.146

\section{INTRODUCTION}

Trigeminal neuralgia, notably known as tic douloroux, is a common disorder in people over 50 years old and more frequently affects females. ${ }^{1}$ Symptoms of trigeminal neuralgia are well recognized, with pain is characterized by recurrent paroxysm onset of pain at the unilateral side of the face, more often distributed into second and third division of the trigeminal nerve. ${ }^{2,3}$ Motions, such as speaking, eating, and any other simple motions or gestures, could trigger and provoke serious pain. ${ }^{4}$ Carbamazepine and other anticonvulsant medication, either used as a solitary medicine or as a part of combination medicine, could improve the quality of pain in terms of patient pain management. Surgical management could also be conducted in recalcitrant case. ${ }^{5}$

The most recent classification distinguishes trigeminal neuralgia into three etiological categories: Classical (generated by neurovascular compression of the fifth cranial nerve), idiopathic (no definitive cause could be found), and secondary (caused by primary neuroglial disease) ${ }^{6}$ Cerebellopontine angle (CPA) tumors are not a frequent cause of secondary trigeminal neuralgia. ${ }^{2,5}$ Diagnosis of trigeminal neuralgia is based primarily on patient history and symptoms. MRI is capable of differentiating etiologies and classifications of trigeminal neuralgia. ${ }^{7}$ According to some authors, only 1 to $9.9 \%$ of trigeminal neuralgia cases occurred due to CPA tumors. ${ }^{8}$ Although the epidermoid lesion is the most frequent cause of trigeminal neuralgia by tumors, the incidence of an epidermoid lesion in intracranial space is not as frequent as the other causes, ranged between 1 to $2 \%$ of all intracranial tumors. ${ }^{9}$

This case study aims to report our satisfactory result regarding the surgical treatment in a female patient with secondary trigeminal neuralgia, accompanied by an epidermoid cyst lesion, which compresses trigeminal nerve root at the right cerebellopontine angle. 


\section{CASE PRESENTATION}

A 47 years old female patient was referred to the Department of Neurosurgery, Margono Soekarjo Regional Hospital, with a significant complaint of exhibiting pain that is increased in intensity and frequency on the right side of the face. The pain, according to the patient, appeared approximately 2 years ago. The pain was characterized as a sudden, sharpshooting pain that lasted only for a few seconds. Pain predominantly affected the fifth cranial nerve's maxillary and mandibular branches, although occasionally, the pain entangled all three branches. The episodes of pain occurred very often, with more episodes happened more frequently in recent times. In the beginning, the pain only occurred three times per week, and the frequency was increasing up to more than five attacks per day in current days before the patient was attended to the hospital. According to the patients, the pain was triggered by simple activities such as washing her face, smiling, chewing, or blowing off her mouth. She had lost $15 \mathrm{~kg}$ last year because of the pain attack, hindering the patient from consuming sufficient intake because of the pain affecting eating motions. There were no other features found associated with this attack, in particular. There was not any associated loss of consciousness, headaches, or visual disturbance. There was a trigger point located in the right zygomatic zone that could inflict pain on physical examination. However, muscles involved in mastication and jaw movement were normal, and their functions were not limited.

The patient had sought treatment at various dental clinics in the first year following the pain with no significant outcome. At a particular clinic, the dentist diagnosed the patient with an infection and damaged teeth on the right maxilla and mandibula. Most of her teeth on the right side were extracted (dental elements 1, 2, 3, 30, and 32).

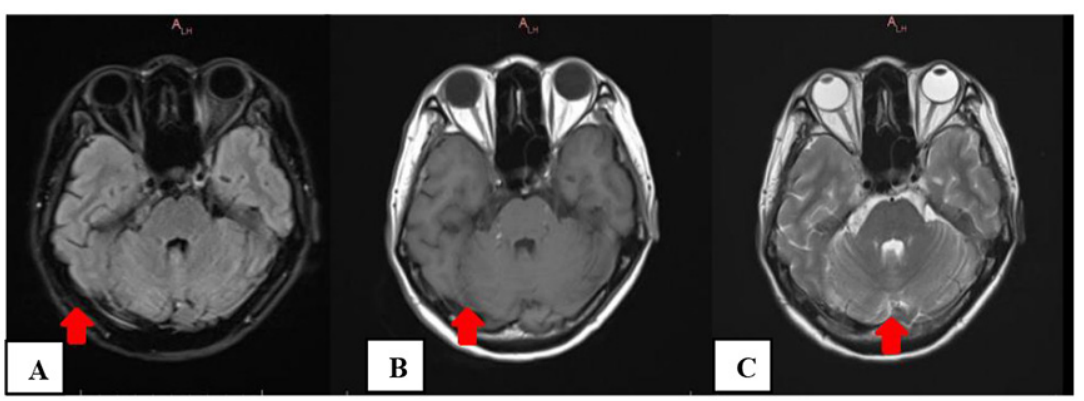

Figure 1. Preoperative (A) axial T1-weighted (B) axial T2-weighted (C) axial T2-FLAIR Magnetic resonance imaging (MRI) images showed a Cerebellopontine angle epidermoid mass (red arrow) compressing the trigeminal nerve.
The patient was referred to the neurologist in a tertiary healthcare hospital after she did not have any significant result, even after receiving medical treatments at the prior dental clinic. The patient was initially receiving carbamazepine single dose of $200 \mathrm{mg}$ and the dosage was increased to 600 mg daily by the neurologist. Still, the patient did not sense any significant benefits after a month of observation with a previously prescribed medicine. The neurologist later prescribed a combination of carbamazepine $200 \mathrm{mg}$ BID and amitriptyline 25 mg daily. This regime resulted only in a transient improvement of her symptoms for about 6 months. Thus, a neurological procedure was recommended by the neurologist and the patient was referred to a neurosurgeon. The patient later received an imaging examination. The cranial Magnetic Resonance Imaging (MRI) of the patient revealed the patient's trigeminal nerve's right branches were compressed by a lesion structured in the right cerebellopontine angle. The lesion appeared as a hypointense coloration in T1 without any significant borderline (Figure 1A) and as a hyperintense coloration in T2 (Figure 1B) and T2 FLAIR (Figure 1C) with some significant differences from brain tissue. MRI study was suggestive of an epidermoid cyst in the right CPA with a lesion size of $15 \times 25 \times 20 \mathrm{~mm}$. Based on these findings, the trigeminal neuralgia diagnosis secondary to the epidermoid cyst located at the cerebellopontine angle was made. Surgical management was planned for this patient, with blood examination suggested normal value on homeostatic.

The patient was taken to the operating table and was administered general anesthesia. The patient was then intubated in a lateral decubitus position without rotating the patient's head. An incision alter was performed by using the retrosigmoid approach after decontamination (Figure 2). Craniotomy and a dural opening were performed to access the right cerebellopontine angle. Identifying any anatomical landmarks was conducted with a microscope until the lesion was found (Figure 3A). The lesion was exposed, with visual details that could be seen in (Figure 3B). Later, the lesion was confirmed as a dermoid cyst or cholesteatoma. After total excision, the operation was finished by dural, skull, and skin closure.

Postoperative complications and any neurological abnormalities were not observed in the patient. The patient was discharged 4 days after a normal postoperative period. Histopathological evaluation revealed an epidermoid cyst with a differential diagnosis of cholesteatoma. It was observed that pain due to trigeminal neuralgia was passed entirely. 


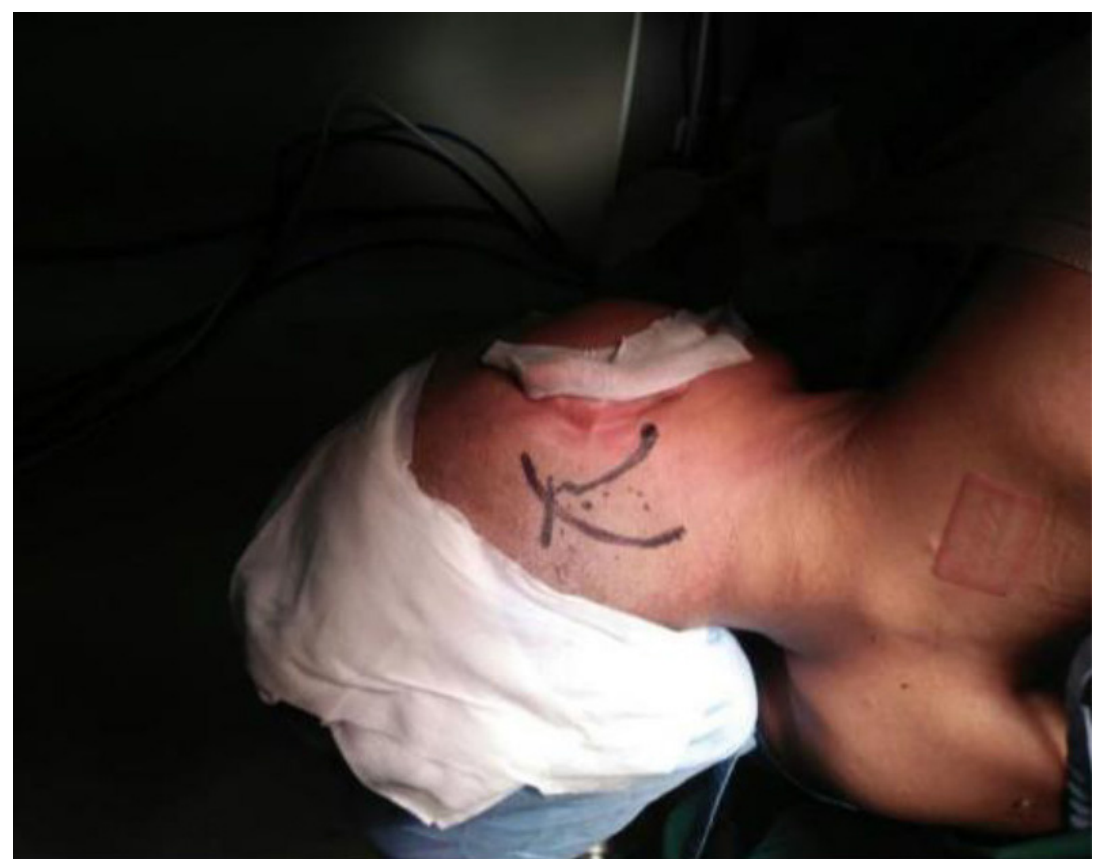

Figure 2. The operating table stayed at the center of the room and the patient was positioned in the lateral decubitus position without rotating the head. Skin drawings showed anatomical landmarks. The straight-full line corresponded to skin incision for the retrosigmoid approach

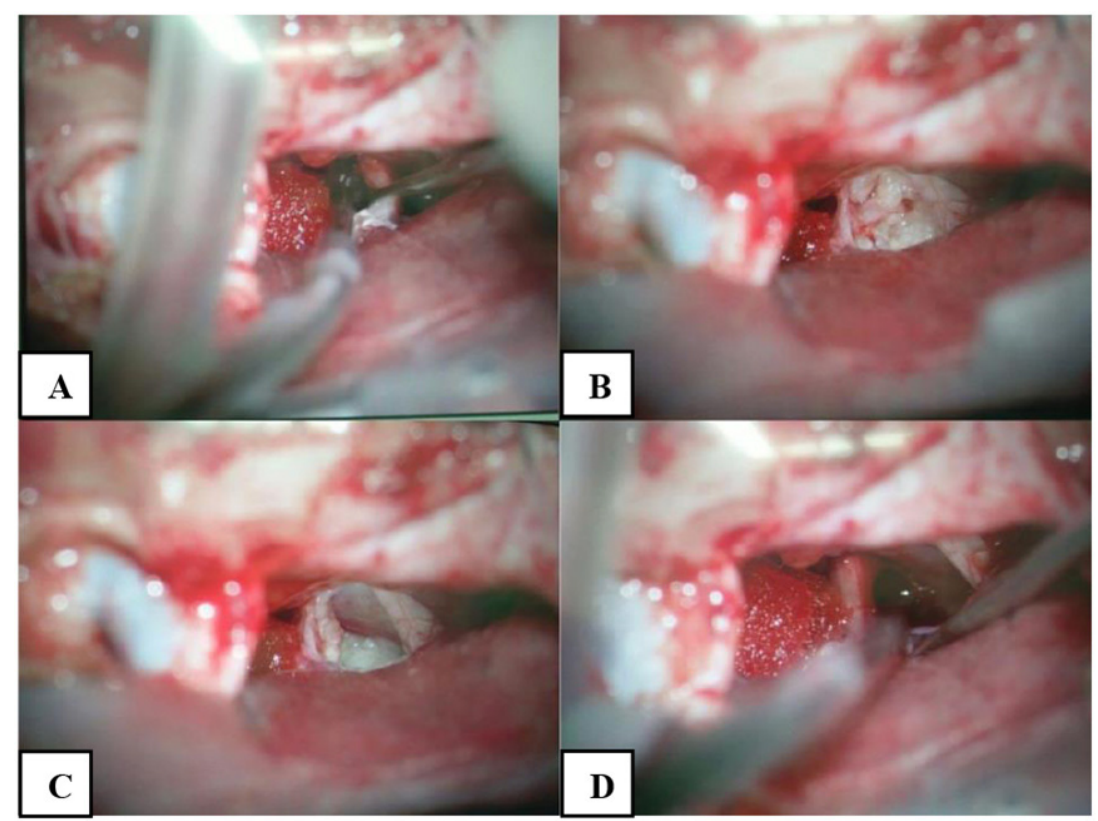

Figure 3. (A) Dissection of VII/VIII complex to avoid arachnoid traction during the trigeminal nerve dissection. (B) After the removal of the arachnoid, the trigeminal nerve was identified, showing a neurovascular conflict. The trigeminal nerve is compressed by a cyst. (C) the cyst was then opened to be evacuated and identified through histopathological examination. (D) total excision lesion. To determine the trigeminal nerve and to make sure the nerve was released from compression

\section{DISCUSSION}

Slow growing benign tumors, including epidermoid cyst, are the most common lesion in intracranial. ${ }^{10}$ An epidermoid cyst contains keratin, cellular debris, and cholesterol covered with stratified squamous epithelium. The term of epidermoid cyst substitute with cholesteatoma., ${ }^{2,3}$ These tumors comprise 1 to $2 \%$ of all intracranial tumors, and most of them are located in the cerebellopontine angle, suprasellar, and ventricle., ${ }^{2,3,5}$ Trigeminal neuralgia is more commonly associated with epidermoid cysts than other intracranial space occupational lesions (SOL) such as meningiomas, acoustic neurinomas, or meningiomas located at the cerebellopontine angle. ${ }^{11}$ As described in recent meta-analysis literature, hearing loss incidence was higher (37.6\%) than trigeminal pain $(29.7 \%) .{ }^{9,12}$ Previous studies also suggest that traumatic brain injury could lead to the trigeminal pain. ${ }^{13,14}$

The pathogenetic mechanism of trigeminal neuralgia in patients with cerebellopontine angle epidermoid cyst remains undefined. Compression throughout the nerve at the root entry zone, enabling any shifting of the trigeminal nerve, compression against a blood vessel at the root entry zone, or both as a combination, has been accepted as the etiology scenarios. ${ }^{12,15}$ Irritation and hyperactivity may be caused by keratins content itself that has been suggested to cause pain in a location where the tumor entirely covers the nerve. ${ }^{12,15}$

Magnetic resonance imaging (MRI) is the most appropriate choice for diagnostic modality for detecting an epidermoid cyst. ${ }^{16}$ However, how to differentiate brain tissue, arachnoid, and epidermoid cysts is sometimes still become a complicated matter. On standard T1- and T2-weighted spinecho MR images, epidermoids often could not be differentiated from cerebrospinal fluid. ${ }^{17,18}$ Fluidattenuated inversion recovery (FLAIR) imaging in MRI can become an optional choice for this case as it can remove the signal of cerebrospinal fluid. ${ }^{12,17,18}$ Tumor removal itself may relieve symptoms, but it must be emphasized that epidermoid tumors commonly cause persistent distortion of the trigeminal nerve, even after a satisfactory resection. Due to the proximity of CP angle epidermoids to the brainstem and cranial nerves, total excision is not possibly conducted in all cases. Caution must be exercised heavily to avoid an overzealous aim for complete removal unless the tumor is easily peeled off. Therefore, severing and removing the adherent tumor capsule and the arachnoid membrane to achieve neural axis straightening is mandatory for the cure and to prevent any recurring pain. ${ }^{17,19}$ 


\section{CONCLUSION}

Trigeminal neuralgia can be seen as a typical symptom in epidermoid cyst cases at the cerebellopontine angle. Epidermoid cyst patients were clinically indistinguishable from patients with trigeminal neuralgia secondary to other causes. The lesion generated trigeminal nerve neuropathy by either compressing the nerve directly or eliciting an inflammatory reaction. MRI scanning was a reasonable choice, in this case, to classify and find the etiology. Our study found that the trigeminal nerve's decompression surgery by total excised tumor leads to a satisfactory result.

\section{CONFLICT OF INTEREST}

There is no competing interest regarding the manuscript.

\section{FUNDING}

None

\section{AUTHOR CONTRIBUTION}

All of the authors equally contribute to the study from the case selection, data gathering, and data analysis until interpreting the case study outcome through publication.

\section{REFERENCES}

1. Zakrzewska JM, Linskey ME. Trigeminal neuralgia. BMJ. 2015;350:h1238.

2. Caldarelli M, Massimi L, Kondageski C, Di Rocco C. Intracranial midline dermoid and epidermoid cysts in children. J Neurosurg. 2004;100(5 Suppl Pediatrics):473-480.

3. Berger MS, Wilson CB. Epidermoid cysts of the posterior fossa. J Neurosurg. 1985;62(2):214-219.

4. Gambeta E, Chichorro JG, Zamponi GW. Trigeminal neuralgia: An overview from pathophysiology to pharmacological treatments. Mol Pain. 2020;16:1744806920901890.

5. Chen S, Ikawa F, Kurisu K, Arita K, Takaba J, Kanou Y. Quantitative MR evaluation of intracranial epidermoid tumors by fast fluid-attenuated inversion recovery imaging and echo-planar diffusion-weighted imaging. AJNR Am J Neuroradiol. 2001;22(6):1089-1096.
6. Eller JL, Raslan AM, Burchiel KJ. Trigeminal neuralgia: definition and classification. Neurosurg Focus. 2005;18(5):E3.

7. Kedarnath NS, Shruthi R. MRI as an essential diagnostic approach for trigeminal neuralgia. J Maxillofac Oral Surg. 2015;14(Suppl 1):462-464.

8. Jamjoom AB, Jamjoom ZA, al-Fehaily M, el-Watidy $S$, al-Moallem M, Nain-Ur-Rahman. Trigeminal neuralgia related to cerebellopontine angle tumors. Neurosurg Rev. 1996;19(4):237-241.

9. Gagliardi FM, Vagnozzi R, Caruso R, Delfini R. Epidermoids of the cerebellopontine angle (cpa): usefulness of CT scan. Acta Neurochir (Wien). 1980;54(3-4):271-281.

10. Kato K, Ujiie H, Higa T, Hayashi M, Kubo O, Okada Y, et al. Clinical presentation of intracranial epidermoids: a surgical series of 20 initial and four recurred cases. Asian J Neurosurg. 2010;5(1):32-40.

11. Guttal KS, Naikmasur VG, Joshi SK, Bathi RJ. Trigeminal neuralgia secondary to epidermoid cyst at the cerebellopontine angle: case report and brief overview. Odontology. 2009;97(1):54-56.

12. Yamakawa K, Shitara N, Genka S, Manaka S, Takakura $\mathrm{K}$. Clinical course and surgical prognosis of 33 cases of intracranial epidermoid tumors. Neurosurgery. 1989;24(4):568-573.

13. Tyburski AL, Cheng L, Assari S, Darvish K, Elliott MB. Frequent mild head injury promotes trigeminal sensitivity concomitant with microglial proliferation, astrocytosis, and increased neuropeptide levels in the trigeminal pain system. J Headache Pain. 2017;18(1):16.

14. Golden N, Mardhika PE, Niryana IW, Sukarya IM, Prabawa IPY. Risk factors and novel prognostic score for predicting the 14-day mortality of severe traumatic brain injury patients. Intisari Sains Medis. 2020;11(3):699-705.

15. Yilmazlar S, Kocaeli H, Cordan T. Brain stem stroke associated with epidermoid tumours: report of two cases. J Neurol Neurosurg Psychiatry. 2004;75(9):1340-1342.

16. Shibata T, Hatori M, Satoh T, Ehara S, Kokubun S. Magnetic resonance imaging features of epidermoid cyst in the extremities. Arch Orthop Trauma Surg. 2003;123(5):239241.

17. Caldarelli M, Colosimo C, Di Rocco C. Intra-axial dermoid/ epidermoid tumors of the brainstem in children. Surg Neurol. 2001;56(2):97-105.

18. Kachhara R, Bhattacharya RN, Radhakrishnan VV. Epidermoid cyst involving the brain stem. Acta Neurochir (Wien). 2000;142(1):97-100.

19. Mallucci CL, Ward V, Carney AS, O'Donoghue GM, Robertson I. Clinical features and outcomes in patients with non-acoustic cerebellopontine angle tumours. J Neurol Neurosurg Psychiatry. 1999;66(6):768-771.

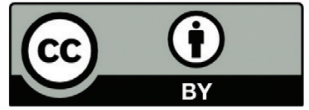

This work is licensed under a Creative Commons Attribution 